\title{
THE ANALYSIS OF MULTIGRID ALGORITHMS FOR PSEUDODIFFERENTIAL OPERATORS OF ORDER MINUS ONE
}

\author{
JAMES H. BRAMBLE, ZBIGNIEW LEYK, AND JOSEPH E. PASCIAK
}

\begin{abstract}
Multigrid algorithms are developed to solve the discrete systems approximating the solutions of operator equations involving pseudodifferential operators of order minus one. Classical multigrid theory deals with the case of differential operators of positive order. The pseudodifferential operator gives rise to a coercive form on $H^{-1 / 2}(\Omega)$. Effective multigrid algorithms are developed for this problem. These algorithms are novel in that they use the inner product on $H^{-1}(\Omega)$ as a base inner product for the multigrid development. We show that the resulting rate of iterative convergence can, at worst, depend linearly on the number of levels in these novel multigrid algorithms. In addition, it is shown that the convergence rate is independent of the number of levels (and unknowns) in the case of a pseudodifferential operator defined by a single-layer potential. Finally, the results of numerical experiments illustrating the theory are presented.
\end{abstract}

\section{INTRODUCTION}

The goal of this paper is to develop a technique for defining and analyzing multigrid algorithms for solving equations which involve discretizations of pseudodifferential operators of negative order. Standard multilevel methods most often apply to discretizations of differential operators of positive order (cf. $[1-4,7-9,16,17])$.

Let $\Omega$ be a polygonal domain in $R^{2}$. For nonnegative real $s$, let $H^{s}(\Omega)$ denote the Sobolev space of real-valued functions with norm $\|\cdot\|_{s}$ (see [14]). In addition, we shall use Sobolev spaces of negative index. For the purpose of this paper, we shall define $H^{-1}(\Omega)$ to be the set of functionals on $H^{1}(\Omega)$ for which the norm

$$
\|v\|_{-1}=\sup _{\phi \in H^{1}(\Omega)} \frac{(v, \phi)}{\|\phi\|_{1}}
$$

is finite. Here $(\cdot, \cdot)$ denotes the inner product in $L^{2}(\Omega)$. For $0<s<1$, the spaces $H^{-s}(\Omega)$ are defined by the real method ( $K$-method) of interpolation

Received by the editor March 12, 1992.

1991 Mathematics Subject Classification. Primary 65N30; Secondary 65F10.

This manuscript has been authored under contract number DE-AC02-76CH00016 with the U.S. Department of Energy. Accordingly, the U.S. Government retains a non-exclusive, royalty-free license to publish or reproduce the published form of this contribution, or allow others to do so, for U.S. Government purposes. This work was also supported in part under the National Science Foundation Grant No. DMS-9007185 and by the U.S. Army Research Office through the Mathematical Sciences Institute, Cornell University. 
[13] between $L^{2}(\Omega)$ and $H^{-1}(\Omega)$. These spaces are Hilbert spaces, and we shall let $\langle\cdot, \cdot\rangle_{-s}$ be the corresponding inner product.

As a canonical example of a pseudodifferential operator of negative order, we consider an operator which is defined in terms of a symmetric bilinear form $\mathscr{V}(\cdot, \cdot)$ on $H^{-1 / 2}(\Omega)$. We will assume that this form satisfies the following coercivity and boundedness inequalities:

$$
C_{0}\|v\|_{-1 / 2}^{2} \leq \mathscr{V}(v, v) \leq C_{1}\|v\|_{-1 / 2}^{2} \quad \text { for all } v \in H^{-1 / 2}(\Omega) .
$$

Here and in the remainder of this paper, $C$ with or without subscripts denotes a generic positive constant which can take on different values in different places. These constants will always be independent of mesh sizes and the number of levels in subsequent multigrid schemes.

Multigrid schemes will be developed in this paper for the efficient solution of the problem: Given a bounded linear functional $F$ on $H^{-1 / 2}(\Omega)$, find $U \in$ $H^{-1 / 2}(\Omega)$ satisfying

$$
\mathscr{V}(U, \theta)=F(\theta) \quad \text { for all } \theta \in H^{-1 / 2}(\Omega) .
$$

This problem has a unique solution by the Riesz Representation Theorem.

The basic philosophy of a multigrid/multilevel algorithm is that simple relaxation schemes can be used to reduce the high eigenvalue components of the errors while a coarser grid problem is used to reduced the smooth components. This works very well in the case of differential operators of positive order since the high eigenvalue components of the differential operator correspond to highly oscillatory components of the error and thus all components are reduced either by smoothing or correction. In contrast, in the case of pseudodifferential operators of negative order, the high eigenvalue component is smooth and thus neither relaxation nor coarse grid correction reduces the oscillatory components.

The solution to the above problem is to use a base inner product which corresponds to a weaker norm than that induced by the form $\mathscr{V}(\cdot, \cdot)$. We use the norm in $H^{-1}(\Omega)$. This effectively changes the relationship between eigenvalues and eigenvectors so that the eigenvectors with large eigenvalues correspond to highly oscillatory components in the eigenspace decomposition.

Smoothers for the multigrid algorithm are developed in terms of discrete inner products which are equivalent to the inner product in $H^{-1}(\Omega)$ on the respective subspaces. The discrete inner products are defined in $\S 3$ in terms of a discretization of a second-order problem. The use of smoothers involving differences for this type of problem was suggested earlier in [12] although no supporting theory was included.

There are three basic theories for providing estimates for V-cycle multigrid algorithms. The first is the so-called "regularity and approximation theory" and provides estimates as long as elliptic regularity results are available for the underlying operator $\mathscr{V}[2,3]$. The second theory requires the weakest hypotheses but gives rise to estimates which depend on the number of levels in the multigrid algorithm [7]. The third theory does not require elliptic regularity and often leads to uniform estimates on the rate of iterative convergence [4].

We prove the conditions required for the application of the "no-regularity" theory of [7] in $\S 4$ provided that (1.1) holds. In $\S 5$, we reduce to the case when 
the form $\mathscr{V}$ is given by the single-layer potential

$$
\mathscr{V}(u, v)=\int_{\Omega} \int_{\Omega} \frac{u\left(s_{1}\right) v\left(s_{2}\right)}{\left|s_{1}-s_{2}\right|} d s_{1} d s_{2}
$$

We will show that (1.1) holds, and hence [7] can be applied. We next apply [4] to develop uniform multigrid convergence estimates in the case when $\Omega$ is a polygonal domain in $R^{2}$. We note that integral operators of positive order are considered in [19].

Finally, the results of numerical experiments are given in $\S 6$. These results are in agreement with the theory of earlier sections.

\section{THE MULTIGRID ALGORITHM}

In this section, we describe the multigrid algorithm following [3]. We assume that we are given a nested sequence of finite-dimensional inner product spaces

$$
\mathscr{M}_{0} \subset \mathscr{M}_{1} \subset \cdots \subset \mathscr{M}_{j} \subset H^{-1 / 2}(\Omega) .
$$

As earlier described, let $\mathscr{V}(\cdot, \cdot)$ be a symmetric positive definite bilinear form on $\mathscr{M}_{j}$ satisfying (1.1). We shall develop multigrid algorithms for computing the solution of the problem: Given a linear functional $F$ on $\mathscr{M}_{j}$, find $u \in \mathscr{M}_{j}$ satisfying

$$
\mathscr{V}(u, \phi)=F(\phi) \quad \text { for all } \phi \in \mathscr{M}_{j}
$$

We describe the multigrid algorithms in terms of certain operators. To this end, we first define $\mathscr{V}_{k}: \mathscr{M}_{k} \mapsto \mathscr{M}_{k}$ by

$$
\left\langle\mathscr{V}_{k} u, v\right\rangle_{-1}=\mathscr{V}(u, v) \quad \text { for all } u, v \in \mathscr{M}_{k} \text {. }
$$

In addition, we define the projectors $\mathscr{P}_{k}: H^{-1}(\Omega) \mapsto \mathscr{M}_{k}$ and $P_{k}: H^{-1 / 2}(\Omega) \mapsto$ $\mathscr{M}_{k}$ by

$$
\left\langle\mathscr{P}_{k} w, \phi\right\rangle_{-1}=\langle w, \phi\rangle_{-1}
$$

and

$$
\mathscr{V}\left(P_{k} w, \phi\right)=\mathscr{V}(w, \phi)
$$

for all $\phi \in \mathscr{M}_{k}$.

The final ingredient in a multigrid algorithm is a sequence of smoothing operators. These operators are defined in terms of additional discrete inner products on $\mathscr{M}_{k}$ which will be denoted $[\cdot, \cdot]_{k}, k=1, \ldots, j$. The smoothing operator $R_{k}: \mathscr{M}_{k} \mapsto \mathscr{M}_{k}$ is then defined by

$$
\left[R_{k} w, \theta\right]_{k}=\frac{1}{\bar{\lambda}_{k}}\langle w, \theta\rangle_{-1} \quad \text { for all } \theta \in \mathscr{M}_{k} .
$$

Here $\bar{\lambda}_{k}$ is an upper bound for the eigenvalue

$$
\lambda_{k}=\sup _{\theta \in \mathscr{M}_{k}} \frac{\mathscr{V}(\theta, \theta)}{[\theta, \theta]_{k}} .
$$

The assumption on $\bar{\lambda}_{k}$ implies that for all $\theta \in \mathscr{M}_{k}$,

$$
\begin{aligned}
\mathscr{V}\left(R_{k} \mathscr{V}_{k} \theta, \theta\right) & \leq \bar{\lambda}_{k}^{1 / 2}\left[R_{k} \mathscr{V}_{k} \theta, R_{k} \mathscr{V}_{k} \theta\right]_{k}^{1 / 2} \mathscr{V}(\theta, \theta)^{1 / 2} \\
& =\mathscr{V}\left(R_{k} \mathscr{V}_{k} \theta, \theta\right)^{1 / 2} \mathscr{V}(\theta, \theta)^{1 / 2}
\end{aligned}
$$


and hence

$$
\mathscr{V}\left(R_{k} \mathscr{V}_{k} \theta, \theta\right) \leq \mathscr{V}(\theta, \theta)
$$

The inequality (2.4) shows that the smoothing operator is properly scaled [3]. In addition, $\bar{\lambda}_{k}$ should be bounded by a fixed multiple of $\lambda_{k}$.

In standard applications, it is often more effective to use smoothers defined by variations of Gauss-Seidel iterative methods [5]. However, in the case of the integral equation application of this paper, Gauss-Seidel smoothing is inappropriate whereas the smoother defined by $(2.3)$ is both computable and theoretically justified.

The multigrid algorithm is defined in terms of a sequence of operators $B_{k}$ : $\mathscr{M}_{k} \mapsto \mathscr{M}_{k}$ which "approximately" invert $\mathscr{V}_{k}$. The following algorithm provides the simplest V-cycle algorithm.

Algorithm 2.1. For $k=0$, set $B_{0}=\mathscr{V}_{0}^{-1}$. For $k>0, B_{k}$ is defined in terms of $B_{k-1}$ as follows: Let $g \in \mathscr{M}_{k}$.

(1) Set

$$
x^{1}=R_{k} g .
$$

(2) Define $x^{2}=x^{1}+q$, where

$$
q=B_{k-1} \mathscr{P}_{k-1}\left(g-\mathscr{V}_{k} x^{1}\right) .
$$

(3) Finally, set

$$
B_{k} g=x^{2}+R_{k}\left(g-\mathscr{V}_{k} x^{2}\right)
$$

The multigrid algorithm is presented this way for theoretical purposes. Even though the use of the inner product on $H^{-1}(\Omega)$ is often not computationally feasible, it is possible to implement the above algorithm in practice provided that the inner products $[\cdot, \cdot]_{k}$ are appropriately defined. These inner products are defined in $\S 3$. They are critical from both the theoretical and implementation points of view. The concrete realization of the algorithm in terms of matrices is also given in $\S 3$.

The first and last step in the above algorithm correspond to smoothing. The second step is the coarser-grid correction step. More general versions of this algorithm involving increased smoothing on the various levels as well as more iterations in the coarser-grid step are defined in the usual way (cf., $[1,3,11$, 15]). Our theory extends to these algorithms as well (cf. [7]).

Typical presentations of the multigrid algorithm directly give rise to an iterative process with a linear reducer. This linear reducer is equal to $I-B_{j} \mathscr{V}_{j}$ (cf. [4]). Thus, the usual multigrid reduction process applied to the problem

$$
\mathscr{V}_{j} v=f
$$

is equivalent to the simple linear preconditioned iterative scheme

$$
v_{i+1}=v_{i}+B_{j}\left(f-\mathscr{V}_{j} v_{i}\right), \quad i=0,1, \ldots,
$$

with $B_{j}$ defined by Algorithm 2.1. Thus, our algorithm is the usual symmetric $\mathrm{V}$-cycle multigrid algorithm described in a notation which is convenient for our analysis. Note that $B_{k}$ is clearly a linear operator for each $k$. 
Nonsymmetric cycling algorithms are defined by avoiding Steps 1 or 3 (cf. [3]). Both the symmetric and nonsymmetric versions are covered by the analysis to be presented. The symmetric operator $B_{j}$ given above can also be used as a preconditioner in, for example, the conjugate gradient algorithm.

\section{THE DISCRETE INNER PRODUCTS}

We define the discrete inner products $[\cdot, \cdot]_{k}$ used in the definition of the smoothing operators in this section. As we shall see, there are two distinct cases depending on whether the functions in $\mathscr{M}_{k}$ are continuous or not. The discrete inner product will always be defined in terms of a difference operator $A_{k}: \mathscr{M}_{k} \mapsto \mathscr{M}_{k}$.

We shall only consider multigrid algorithms for finite element approximations to (1.2) in this paper. Because the form $\mathscr{V}(\cdot, \cdot)$ is so weak, the finite element approximation subspaces need only be in $H^{-1 / 2}(\Omega)$. However, for simplicity, we shall first consider the case when $\mathscr{M}_{k}$ consists of continuous piecewise linear finite element functions.

To this end, we start with a coarse triangulation $\left\{\tau_{0}^{i}\right\}$ of $\Omega$. Assuming that $\left\{\tau_{k-1}^{i}\right\}$ has been defined, the finer triangulation $\left\{\tau_{k}^{i}\right\}$ is defined by breaking each triangle in $\left\{\tau_{k-1}^{i}\right\}$ into four by connecting the midpoints of the sides. The space $\mathscr{M}_{k}$, for $k=0, \ldots, j$, is defined to be the set of functions which are piecewise linear with respect to $\left\{\tau_{k}^{i}\right\}$ and continuous on $\Omega$.

To avoid the inversion of Gram matrices in the multigrid implementation, we next consider a diagonal inner product approximating the $L^{2}(\Omega)$ inner product on the subspace. Let $(\cdot, \cdot)_{k}$ be defined for $v \in \mathscr{M}_{k}$ by

$$
(v, v)_{k}=\frac{1}{3} \sum_{\tau_{k}^{i}}\left|\tau_{k}^{i}\right|\left[v\left(x_{k}^{i, 1}\right)^{2}+v\left(x_{k}^{i, 2}\right)^{2}+v\left(x_{k}^{i, 3}\right)^{2}\right] .
$$

Here, $\left|\tau_{k}^{i}\right|$ denotes area of $\tau_{k}^{i}$ and $\left\{x_{k}^{i, l}\right\}, l=1,2,3$, denotes the vertices of $\tau_{k}^{i}$. It is known that

$$
\left|(v, w)_{k}-(v, w)\right| \leq C h_{k}\|v\|\|w\|_{1} \quad \text { for all } v \in \mathscr{M}_{k}, w \in \mathscr{M}_{k},
$$

where $h_{k}\left(h_{k}=2^{-k} h_{0}\right)$ is the mesh size of the $k$ th mesh. It is also immediate that

$$
c(v, v)_{k} \leq(v, v) \leq C(v, v)_{k} \quad \text { for all } v \in \mathscr{M}_{k} .
$$

The discrete form $[\cdot, \cdot]_{k}$ is defined in terms of the finite element discretization of a second-order problem. For $v, w$ in $H^{1}(\Omega)$, let

$$
A(v, w)=\int_{\Omega}(\nabla v \cdot \nabla w+v w) d x .
$$

Clearly, $A(\cdot, \cdot)$ is symmetric and positive definite on $H^{1}(\Omega)$ and

$$
\|u\|_{1}=A(u, u)^{1 / 2} \quad \text { for all } u \in H^{1}(\Omega) .
$$

For $k=0, \ldots, j$, let the operators $A_{k}: \mathscr{M}_{k} \mapsto \mathscr{M}_{k}$ be defined by

$$
\left(A_{k} w, \phi\right)_{k}=A(w, \phi) \quad \text { for all } w, \phi \in \mathscr{M}_{k} \text {. }
$$


The operator $A_{k}$ is clearly symmetric (in both the $A(\cdot, \cdot)$ and $(\cdot, \cdot)_{k}$ inner products) and positive definite. In this case, the discrete inner product used in the multigrid algorithm is defined by

$$
[u, v]_{k}=\left(A_{k}^{-1} u, v\right)_{k} \quad \text { for all } u, v \in \mathscr{M}_{k} .
$$

As we shall see later, the implementation of the multigrid algorithm using this discrete inner product only requires the evaluation of the action of $A_{k}$ (not $\left.A_{k}^{-1}\right)$. The next lemma shows that the norm corresponding to this inner product is uniformly equivalent to the norm in $H^{-1}(\Omega)$.

Lemma 3.1. Let $[\cdot, \cdot]_{k}$ be defined by (3.5). There exist positive constants $C_{0}, C_{1}$ which are independent of $j$ and satisfy

$$
C_{0}\|v\|_{-1}^{2} \leq[v, v]_{k} \leq C_{1}\|v\|_{-1}^{2} .
$$

The inequalities (3.6) hold for all $v \in \mathscr{M}_{k}$ and $k=0, \ldots, j$.

Proof. Let $Q_{k}$ denote the $L^{2}(\Omega)$ orthogonal projector onto $\mathscr{M}_{k}$. It is well known that (see, for example, [10])

$$
\left\|\left(I-Q_{k}\right) w\right\| \leq C h_{k}\|w\|_{1}, \quad\left\|Q_{k} w\right\|_{1} \leq C\|w\|_{1},
$$

for all $w \in H^{1}(\Omega)$. Moreover, it easily follows from (3.7) and the fact that $\mathscr{M}_{k} \subset H^{1}(\Omega)$, that

$$
C_{2}\|w\|_{-1}^{2} \leq \sup _{\phi \in \mathscr{M}_{k}} \frac{(w, \phi)^{2}}{A(\phi, \phi)} \leq\|w\|_{-1}^{2} \quad \text { for all } w \in \mathscr{M}_{k} .
$$

The constant $C_{2}$ is independent of $k$. Let $v$ be in $\mathscr{M}_{k}$. We note that

$$
[v, v]_{k}=\left(A_{k}^{-1} v, v\right)_{k}=\sup _{\phi \in \mathscr{M}_{k}} \frac{\left(v, A_{k}^{-1 / 2} \phi\right)_{k}^{2}}{(\phi, \phi)_{k}}=\sup _{\theta \in \mathscr{M}_{k}} \frac{(v, \theta)_{k}^{2}}{A(\theta, \theta)} .
$$

Using the well-known inverse property,

$$
A(\phi, \phi) \leq c h_{k}^{-2}\|\phi\|^{2} \quad \text { for all } \phi \in \mathscr{M}_{k},
$$

and (3.8) implies

$$
\|v\|^{2}=\sup _{\phi \in \mathscr{M}_{k}} \frac{(v, \phi)^{2}}{\|\phi\|^{2}} \leq C h_{k}^{-2} \sup _{\phi \in \mathscr{M}_{k}} \frac{(v, \phi)^{2}}{A(\phi, \phi)} \leq C h_{k}^{-2}\|v\|_{-1}^{2} .
$$

The same argument and (3.2) gives that

$$
\|v\|^{2} \leq C h_{k}^{-2} \sup _{\phi \in \mathscr{M}_{k}} \frac{(v, \phi)_{k}^{2}}{A(\phi, \phi)} .
$$

Applying (3.1) and (3.10) gives

$$
\begin{aligned}
\left|(v, \theta)_{k}\right| & \leq\left|(v, \theta)_{k}-(v, \theta)\right|+|(v, \theta)| \\
& \leq C h_{k}\|v\|\|\theta\|_{1}+\|v\|_{-1}\|\theta\|_{1} \leq C\|v\|_{-1}\|\theta\|_{1} .
\end{aligned}
$$

Combining (3.9) and (3.12) proves the upper inequality of (3.6).

For the lower inequality, because of (3.8), we need only show that

$$
\sup _{\phi \in \mathscr{M}_{k}} \frac{(v, \phi)^{2}}{A(\phi, \phi)} \leq C \sup _{\phi \in \mathscr{M}_{k}} \frac{(v, \phi)_{k}^{2}}{A(\phi, \phi)} .
$$


Applying (3.1) gives

$$
\begin{aligned}
|(v, \theta)| & \leq\left|(v, \theta)-(v, \theta)_{k}\right|+\left|(v, \theta)_{k}\right| \\
& \leq C h_{k}\|v\|\|\theta\|_{1}+\left|(v, \theta)_{k}\right| .
\end{aligned}
$$

By combining (3.11) and (3.14), the inequality (3.13) follows. This completes the proof of the lemma.

We next consider the case when the subspaces are discontinuous functions. In this case we assume for simplicity that $\Omega$ is a rectangle in $R^{2}$. We divide this rectangle into a rectangular mesh to define the partitioning $\left\{\tau_{0}^{i}\right\}$. Subsequently finer meshes are defined by mathematical induction. Given $\left\{\tau_{k-1}^{i}\right\}$, the rectangles of $\left\{\tau_{k}^{i}\right\}$ are defined by breaking each rectangle in $\left\{\tau_{k-1}^{i}\right\}$ into four congruent smaller rectangles. Functions in $\mathscr{M}_{k}$ are defined to be piecewise constant with respect to the mesh $\left\{\tau_{k}^{i}\right\}$.

In this application, we do not require the introduction of a discrete $L^{2}(\Omega)$ inner product, since the Gram matrix for $\mathscr{M}_{k}$ (with the natural basis) is already diagonal. The discrete operators $A_{k}$ are defined directly in terms of differences. Functions in $\mathscr{M}_{k}$ are determined by doubly indexed arrays of coefficients $\left\{c_{i j}\right\}$, $(i, j) \in \mathscr{N}_{k}$. The set $\mathscr{N}_{k}$ consists of the indices labeling the rectangles in $\left\{\tau_{k}^{i}\right\}$. $A_{k}$ is defined to be the symmetric difference operator with diagonal

$$
\left(A_{k} c, c\right)=h_{k}^{2} \sum_{(i, j) \in \mathscr{N}_{k}} c_{i j}^{2}+\sum\left(c_{i j}-c_{i, j+1}\right)^{2}+\sum\left(c_{i j}-c_{i+1, j}\right)^{2}
$$

Terms are included in the last two sums above if and only if both of the coefficients' indices are in $\mathscr{N}_{k}$. Note that $A_{k}$ is the five-point difference operator with appropriate modification near the boundary. We define $[\cdot, \cdot]_{k}$ by

$$
[v, w]_{k}=\left(A_{k}^{-1} v, w\right) \quad \text { for all } v, w \in \mathscr{M}_{k} \text {. }
$$

We then have the following lemma.

Lemma 3.2. Let $A_{k}$ be defined by (3.15). Then (3.6) holds for $[\cdot, \cdot]_{k}$ defined by (3.16).

Proof. Let $v$ be an arbitrary function in $\mathscr{M}_{k}$. We first note that

$$
[v, v]_{k}=\left(A_{k}^{-1} v, v\right)=\sup _{\phi \in \mathscr{M}_{k}} \frac{(v, \phi)^{2}}{\left(A_{k} \phi, \phi\right)} .
$$

We again let $Q_{k}$ denote the $L^{2}(\Omega)$ orthogonal projection onto $\mathscr{M}_{k}$. It is easy to check that

$$
\left(A_{k} Q_{k} \theta, Q_{k} \theta\right) \leq C\|\theta\|_{1}^{2} \quad \text { for all } \theta \in H^{1}(\Omega) .
$$

Consequently,

$$
\begin{aligned}
\|v\|_{-1}^{2} & =\sup _{\theta \in H^{1}(\Omega)} \frac{\left(v, Q_{k} \theta\right)^{2}}{\|\theta\|_{1}^{2}} \\
& \leq C \sup _{\theta \in H^{1}(\Omega)} \frac{\left(v, Q_{k} \theta\right)^{2}}{\left(A_{k} Q_{k} \theta, Q_{k} \theta\right)}=C\left(A_{k}^{-1} v, v\right) .
\end{aligned}
$$

Let $\phi$ be in $\mathscr{M}_{k}$. We define a shifted rectangular mesh $\left\{\tilde{\tau}_{k}^{i}\right\}$ by connecting the centers of the original rectangles and let $\Omega_{k}$ denote the union of the shifted 


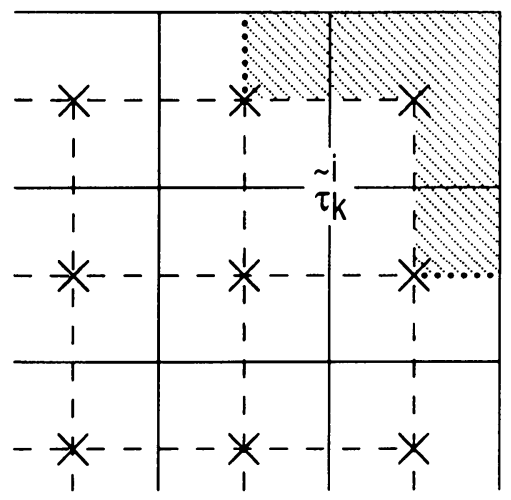

(a)

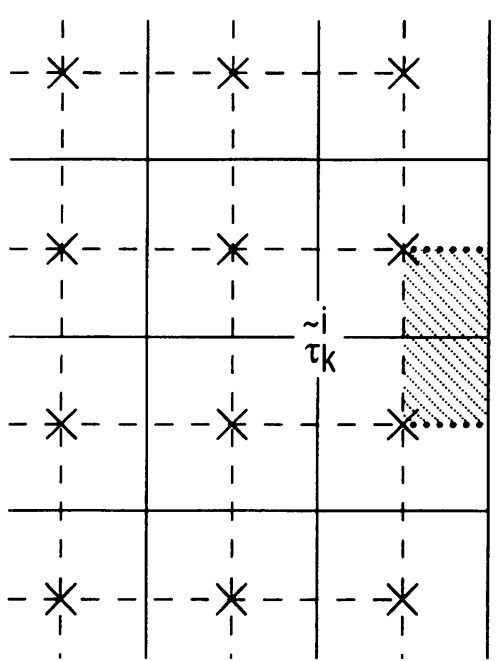

(b)

FIGURE 1. Local extension regions

rectangles contained in $\Omega$ (see the rectangular mesh formed by the dashed lines in Figure 1). Note that $\Omega_{k}$ differs from $\Omega$ by a strip of width $h_{k} / 2$. Let $\Phi$ denote the function which is continuous on $\Omega_{k}$, piecewise bilinear with respect to the shifted rectangles, and interpolates $\phi$ on the vertices of the shifted rectangles (these vertices are labelled $\times$ in Figure 1). We extend $\Phi$ to all of $\Omega$ in a piecewise bilinear fashion. For example, the bilinear function $\Phi$ on the shifted triangle $\tilde{\tau}_{k}^{i}$ in Figure 1 is extended bilinearly into the shaded region. Figure 1 (a) illustrates the case of a corner of $\Omega$ while Figure 1 (b) illustrates the case of an edge of $\Omega$. This extension is also denoted by $\Phi$. It is easy to see that $\Phi \in H^{1}(\Omega)$ and furthermore, it is elementary to see that

$$
c\left(A_{k} \phi, \phi\right) \leq\|\Phi\|_{1}^{2} \leq C\left(A_{k} \phi, \phi\right), \quad\|\phi-\Phi\| \leq C h_{k}\|\Phi\|_{1},
$$

for all $\phi \in \mathscr{M}_{k}$.

We now prove the upper bound of (3.6). By (3.18),

$$
\begin{aligned}
|(v, \phi)| & \leq|(v, \phi-\Phi)|+|(v, \Phi)| \\
& \leq C\left(h_{k}\|v\|+\|v\|_{-1}\right)\|\Phi\|_{1} \leq C\left(h_{k}\|v\|+\|v\|_{-1}\right)\left(A_{k} \phi, \phi\right)^{1 / 2} .
\end{aligned}
$$

Thus, using (3.17), the upper inequality of (3.6) will follow if we prove that

$$
\|v\| \leq C h_{k}^{-1}\|v\|_{-1} .
$$

For any $\theta \in \mathscr{M}_{k}$, there exists a function $\Theta \in H^{1}(\Omega)$ which has the same average values as $\theta$ on the rectangles of $\left\{\tau_{k}^{i}\right\}$ and satisfies

$$
\|\Theta\|_{1} \leq C h_{k}^{-1}\|\theta\| \text {. }
$$

In fact, $\Theta$ can be constructed by taking linear combinations of smooth functions supported on the rectangles of $\left\{\tau_{k}^{i}\right\}$. Thus,

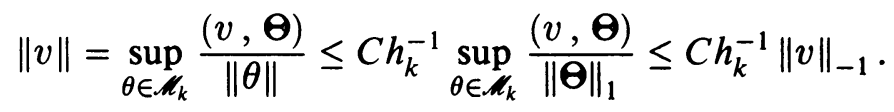


This proves (3.20) and hence completes the proof of the lemma.

We now present the matrix form of Algorithm 2.1, since this elucidates its computational implementation. A common notation for the two cases can be developed if we define $(\cdot, \cdot)_{k}=(\cdot, \cdot)$ in the case when $\mathscr{M}_{k}$ consists of discontinuous constants.

We shall use a bar for denoting matrices and vectors. Let $n_{k}=\operatorname{dim}\left(\mathscr{M}_{k}\right)$ and $\left\{\phi_{k}^{l}\right\}_{l=1}^{n_{k}}$ be the natural basis functions for $\mathscr{M}_{k}$. Note that by scaling the basis functions, we may assume that $\left(\phi_{k}^{i}, \phi_{k}^{l}\right)_{k}=\delta_{i l}$, where $\delta_{i l}$ is the Kronecker Delta. Let

$$
\bar{A}_{k}=\left\{\left(A_{k} \phi_{k}^{i}, \phi_{k}^{l}\right)_{k}\right\}_{i, l=1}^{n_{k}}
$$

and

$$
\overline{\mathscr{V}}_{k}=\left\{\mathscr{V}\left(\phi_{k}^{i}, \phi_{k}^{l}\right)\right\}_{i, l=1}^{n_{k}} .
$$

In addition, let the matrix $\bar{C}_{k}=\left\{c_{k}^{i l}\right\}$ be defined from the coefficients $\left\{c_{k}^{i l}\right\}$ satisfying $\phi_{k}^{i}=\sum_{l=1}^{n_{k+1}} c_{k}^{i l} \phi_{k+1}^{l}$.

In terms of matrices, $(2.1)$ can be represented

$$
\overline{\mathscr{V}}_{j} \bar{u}=\bar{F} \text {. }
$$

Here, $\bar{u}$ is the vector of coefficients in the expansion of the function $u$ in the basis $\left\{\phi_{j}^{i}\right\}$, and $\bar{F}$ is the vector $\left\{F\left(\phi_{j}^{i}\right)\right\}$.

We consider the matrix operator $\bar{B}_{j}$ defined by the following algorithm. As we shall see in the subsequent proposition, this algorithm provides a concrete realization of the operator $B_{j}$.

Algorithm 3.1 (Matrix form of Algorithm 2.1).

Set $\bar{B}_{0}=\overline{\mathscr{V}}_{0}^{-1}$. Assume that $\bar{B}_{k-1}$ has been defined and define $\bar{B}_{k} \bar{G}$ for $\bar{G} \in R^{n_{k}}$ as follows:

(1) Set

$$
\bar{x}^{1}=\bar{\lambda}_{k}^{-1} \overline{A_{k}} \bar{G} .
$$

(2) Define $\bar{x}^{2}=\bar{x}^{1}+\bar{C}_{k-1}^{t} \bar{q}$, where

$$
\bar{q}=\bar{B}_{k-1} \bar{C}_{k-1}\left(\bar{G}-\overline{\mathscr{V}}_{k} \bar{x}^{1}\right) .
$$

(3) Finally, set

$$
\bar{B}_{k} \bar{G}=\bar{x}^{2}+\bar{\lambda}_{k}^{-1} \bar{A}_{k}\left(\bar{G}-\overline{\mathscr{V}}_{k} \bar{x}^{2}\right) .
$$

Proposition 3.1. Let $v \in \mathscr{M}_{k}$ and $\bar{v}$ denote the vector of coefficients for the expansion of the function $v$ in the basis $\left\{\phi_{k}^{i}\right\}$. Then, $\bar{B}_{k} \overline{\mathscr{V}}_{k} v$ is the vector of coefficients for the expansion of the function $B_{k} \mathscr{V}_{k} v$.

Proof. The proof is by induction. The result is obvious for $k=0$. Let $k$ be greater than zero and let $v$ and $\bar{v}$ be as above. We consider applying Algorithm 2.1 to $\mathscr{V}_{k} v$ and Algorithm 3.1 to $\mathscr{\mathscr { V }}_{k} \bar{v}$. In the first step of Algorithm 2.1, we compute the function $x^{1}$ satisfying

$$
\left[x^{1}, \theta\right]_{k}=\left(A_{k}^{-1} x^{1}, \theta\right)_{k}=\bar{\lambda}_{k}^{-1} \mathscr{V}(v, \theta) \quad \text { for all } \theta \in \mathscr{M}_{k} .
$$

In contrast, $\bar{G}=\overline{\mathscr{V}}_{k} \bar{v}$ in Algorithm 3.1. Let $G \in \mathscr{M}_{k}$ satisfy

$$
(G, \theta)_{k}=\mathscr{V}(v, \theta) \quad \text { for all } \theta \in \mathscr{M}_{k} \text {. }
$$


Note that $\bar{G}$ is the vector of coefficients for the expansion of $G$ in the basis for $\mathscr{M}_{k}$. Combining (3.24) and (3.25) and changing variables gives

$$
\left(x^{1}, \phi_{k}^{i}\right)_{k}=\bar{\lambda}_{k}^{-1}\left(A_{k} G, \phi_{k}^{i}\right)_{k}, \quad i=1, \ldots, n_{k} .
$$

This means that the coefficients of $x^{1}$ (denoted by $\bar{x}^{1}$ ) satisfy

$$
\bar{x}^{1}=\bar{\lambda}_{k}^{-1} \overline{A_{k} G}=\bar{\lambda}_{k}^{-1} \bar{A}_{k} \bar{G} \text {. }
$$

This coincides with the first step of Algorithm 3.1.

To compare the results of the second steps of the algorithms, we note that the previous conclusion immediately implies that $\bar{G}-\overline{\mathscr{V}}_{k} \bar{x}^{1}=\overline{\mathscr{V}}_{k}\left(\bar{v}-\bar{x}^{1}\right)$ equals the vector $\left\{\mathscr{V}\left(v-x^{1}, \phi_{k}^{i}\right)\right\}$. Consequently, $\bar{C}_{k-1}\left(\bar{G}-\overline{\mathscr{V}}_{k} \bar{x}^{1}\right)$ is the vector $\left\{\mathscr{V}\left(v-x^{1}, \phi_{k-1}^{i}\right)\right\}$. However,

$$
\begin{aligned}
\mathscr{V}\left(v-x^{1}, \phi_{k-1}^{i}\right) & =\left\langle\mathscr{P}_{k-1} \mathscr{V}_{k}\left(v-x^{1}\right), \phi_{k-1}^{i}\right\rangle_{-1} \\
& =\left\langle\mathscr{V}_{k-1} P_{k-1}\left(v-x^{1}\right), \phi_{k-1}^{i}\right\rangle_{-1} .
\end{aligned}
$$

Note that the right-hand side of (3.26) is $\overline{\mathscr{V}}_{k-1}$ applied to the coefficients of $P_{k-1}\left(v-x^{1}\right)$ expanded in the basis for $\mathscr{M}_{k-1}$. Thus, by the induction hypothesis, $\bar{q}$ in Algorithm 3.1 is the vector of coefficients (with respect to the basis for $\mathscr{M}_{k-1}$ ) of the expansion of

$$
B_{k-1} \mathscr{V}_{k-1} P_{k-1}\left(v-x^{1}\right)=q .
$$

Consequently, $\bar{x}^{2}$ defined by Algorithm 3.1 gives the coefficients of $x^{2}$ defined by Algorithm 2.1 .

The proof that the final step of Algorithm 3.1 results in the coefficients of the function developed in the final step of Algorithm 2.1 is similar. This completes the proof of the proposition.

Remark 3.1. The proposition immediately implies that for all $v \in \mathscr{M}_{j}$,

$$
\left(\overline{\mathscr{V}}_{j}\left(I-\bar{B}_{j} \overline{\mathscr{V}}_{j}\right) \bar{v}\right) \cdot \bar{v}=\mathscr{V}\left(\left(I-B_{j} \mathscr{V}_{j}\right) v, v\right)
$$

and

$$
\left(\mathscr{V}_{j} \bar{v}\right) \cdot \bar{v}=\mathscr{V}(v, v) .
$$

Thus, contraction estimates for $I-B_{j} \mathscr{V}_{j}$ and estimates on the condition number $\kappa\left(B_{j} \mathscr{V}_{j}\right)$ lead to the same results for their matrix counterparts.

\section{4. "NO-REGULARITY" MULTIGRID ANALYSIS}

We apply the theory of [7] to develop a convergence theory for Algorithm 2.1. To do this, we need operators $\mathscr{Q}_{k}: \mathscr{M}_{j} \mapsto \mathscr{M}_{k}$ with $\mathscr{Q}_{j}=I$ and satisfying

$$
\begin{aligned}
\left\|\left(\mathscr{Q}_{k}-\mathscr{Q}_{k-1}\right) u\right\|_{-1}^{2} & \leq C_{2} \lambda_{k}^{-1} \mathscr{V}(u, u) \text { for } k=1, \ldots, j, \\
\mathscr{V}\left(\mathscr{Q}_{k} u, \mathscr{Q}_{k} u\right) & \leq C_{3} \mathscr{V}(u, u) \text { for } k=0, \ldots, j-1 .
\end{aligned}
$$

The inequalities (4.1) and (4.2) hold for all $u \in \mathscr{M}_{j}$.

Lemma 4.1. Let $\mathscr{M}_{k}$ consist of continuous piecewise linear functions on triangles as described in the first application of the previous section, and set $\mathscr{Q}_{k}=Q_{k}$. Then (4.1) and (4.2) hold with constants $C_{2}$ and $C_{3}$ which are independent of $j$.

Proof. The inequalities (3.7) and duality immediately imply that

$$
\begin{aligned}
\left\|\left(I-Q_{k}\right) w\right\|_{-1} & \leq C h_{k}\|w\| & & \text { for all } w \in L^{2}(\Omega), \\
\left\|Q_{k} w\right\|_{-1} & \leq C\|w\|_{-1} & & \text { for all } w \in H^{-1}(\Omega) .
\end{aligned}
$$


Applying the real method of interpolation gives

$$
\left\|\left(I-Q_{k}\right) w\right\|_{-1} \leq c h_{k}^{1 / 2}\|w\|_{-1 / 2} \quad \text { for all } w \in H^{-1 / 2}(\Omega) .
$$

Let $v$ be an arbitrary function in $\mathscr{M}_{j}$. Applying (1.1) gives that

$$
\begin{aligned}
\left\|\left(Q_{k}-Q_{k-1}\right) v\right\|_{-1}^{2} & \leq 2\left\|\left(I-Q_{k}\right) v\right\|_{-1}^{2}+2\left\|\left(I-Q_{k-1}\right) v\right\|_{-1}^{2} \\
& \leq C h_{k}\|v\|_{-1 / 2}^{2} \leq C h_{k} \mathscr{V}(v, v) .
\end{aligned}
$$

By interpolation and (4.3),

$$
\begin{aligned}
\mathscr{V}\left(Q_{k} w, Q_{k} w\right) & \leq C\left\|Q_{k} w\right\|_{-1 / 2}^{2} \\
& \leq C\|w\|_{-1 / 2}^{2} \leq C \mathscr{V}(w, w) \quad \text { for all } w \in H^{-1 / 2}(\Omega) .
\end{aligned}
$$

To complete the proof, we need only show that

By definition,

$$
h_{k} \leq C \lambda_{k}^{-1} \text {. }
$$

$$
\lambda_{k}=\sup _{\phi \in \mathscr{M}_{k}} \frac{\mathscr{V}(\phi, \phi)}{[\phi, \phi]_{k}} \leq C \sup _{\phi \in \mathscr{M}_{k}} \frac{\|\phi\|_{-1 / 2}^{2}}{\|\phi\|_{-1}^{2}} .
$$

By (3.8) and (3.10), for $\phi \in \mathscr{M}_{k}$,

$$
\|\phi\|_{-1 / 2}^{2} \leq C\|\phi\|\|\phi\|_{-1} \leq C h_{k}^{-1}\|\phi\|_{-1}^{2} \text {. }
$$

This completes the proof of the lemma.

Lemma 4.2. Let $\mathscr{M}_{k}$ consist of discontinuous piecewise constant functions on rectangles as described in the second application of the previous section, and set $\mathscr{Q}_{k}=\mathscr{P}_{k}$. Then, (4.1) and (4.2) hold with constants $C_{2}$ and $C_{3}$ which are independent of $j$.

Proof. As in the proof of Lemma 4.1, it follows from (3.22) that $h_{k} \leq C \lambda_{k}^{-1}$. In addition, for $w \in L^{2}(\Omega)$,

$$
\left\|\left(I-Q_{k}\right) w\right\|_{-1}=\sup _{\theta \in H^{\prime}(\Omega)} \frac{\left(w,\left(I-Q_{k}\right) \theta\right)}{\|\theta\|_{1}} \leq C h_{k}\|w\| .
$$

Since $\left(I-\mathscr{Q}_{k}\right) w$ is the minimizer,

$$
\left\|\left(I-\mathscr{Q}_{k}\right) w\right\|_{-1} \leq C h_{k}\|w\| .
$$

The operator $\left(I-\mathscr{Q}_{k}\right)$ is clearly bounded from $H^{-1}(\Omega)$ into $H^{-1}(\Omega)$, and hence by interpolation,

$$
\left\|\left(I-\mathscr{Q}_{k}\right) w\right\|_{-1} \leq C h_{k}^{1 / 2}\|w\|_{-1 / 2} \quad \text { for all } w \in H^{-1 / 2}(\Omega) .
$$

Inequality (4.1) follows from (1.1).

For inequality (4.2), we first note that by (3.22),

$$
\left\|\left(\mathscr{Q}_{k}-Q_{k}\right) w\right\| \leq C h_{k}^{-1}\left\|\left(\mathscr{Q}_{k}-Q_{k}\right) w\right\|_{-1} \leq C\|w\| \quad \text { for all } w \in L^{2}(\Omega) \text {. }
$$

Consequently, $\mathscr{Q}_{k}$ is bounded both as an operator on $L^{2}(\Omega) \mapsto L^{2}(\Omega)$ as well as an operator on $H^{-1}(\Omega) \mapsto H^{-1}(\Omega)$. By interpolation,

$$
\left\|\mathscr{Q}_{k} w\right\|_{-1 / 2} \leq C\|w\|_{-1 / 2} \quad \text { for all } w \in H^{-1 / 2}(\Omega) \text {. }
$$

Inequality (4.2) now follows from (1.1). This completes the proof of the lemma.

The following theorem is a consequence of [7] and Lemmas 4.1 and 4.2. 
Theorem 4.1. Let $\mathscr{M}_{k}$ be as in $\S 3$. There exists a constant $C$ not depending on $j$ such that

$$
0 \leq \mathscr{V}\left(\left(I-B_{j} \mathscr{V}_{j}\right) u, u\right) \leq \delta_{j} \mathscr{V}(u, u) \quad \text { for all } u \in \mathscr{M}_{j},
$$

where

$$
\delta_{j}=1-(C j)^{-1}
$$

In terms of matrices,

$$
0 \leq\left(\overline{\mathscr{V}}_{j}\left(I-\bar{B}_{j} \overline{\mathscr{V}}_{j}\right) \bar{v}\right) \cdot \bar{v} \leq \delta_{j}\left(\overline{\mathscr{V}}_{j} \bar{v}\right) \cdot \bar{v} \quad \text { for all } \bar{v} \in R^{n_{k}} .
$$

Proof. We need only verify that the smoothing operators satisfy appropriate conditions. The appropriate upper bound for the smoothing operator is given by (2.4). By the definition of $R_{k}$ and Lemmas 3.1 and 3.2, for $w \in \mathscr{M}_{k}$,

$$
\|w\|_{-1}^{2}=\bar{\lambda}_{k}\left[R_{k}^{1 / 2} w, R_{k}^{1 / 2} w\right]_{k} \leq c \lambda_{k}\left\langle R_{k} w, w\right\rangle_{-1} .
$$

This provides the appropriate lower bound and completes the proof of the theorem.

Remark 4.1. The above theorem provides an estimate for the contraction associated with the linear preconditioned iteration

$$
\bar{u}_{i+1}=\bar{u}_{i}+\bar{B}_{j}\left(\bar{F}-\overline{\mathscr{V}}_{j} \bar{u}_{i}\right), \quad i=0,1, \ldots .
$$

In fact, the error $\bar{e}_{i}=\bar{u}-\bar{u}_{i}$ satisfies

$$
\left(\overline{\mathscr{V}}_{j} \bar{e}_{i}\right) \cdot \bar{e}_{i} \leq \delta_{j}^{2 i}\left(\overline{\mathscr{V}}_{j} \bar{e}_{0}\right) \cdot \bar{e}_{0} .
$$

In addition, the theorem implies that the condition number $\kappa\left(\bar{B}_{j} \overline{\mathscr{V}}_{j}\right)$ is bounded by $C j$. Such a bound implies that the corresponding preconditioned conjugate gradient iteration also converges rapidly.

Remark 4.2. There is no problem extending the results of this section to the case when $\Omega$ consists of a union of polygonal faces and forms the boundary of a domain in $R^{3}$.

\section{THE CASE OF A SINGLE-LAYER POTENTIAL}

In this section, we consider the case when the form $\mathscr{V}$ is defined from the single-layer potential (1.3). It is first shown that (1.1) holds. Next, conditions for the application of the theory of [4] are verified and lead to iterative convergence estimates for the multigrid algorithm which are independent of the number of levels.

Let $\mathscr{S}$ be a smooth boundary of a domain in $R^{3}$. It was shown in [18] that

$$
C_{0}\|v\|_{-1 / 2, \mathscr{S}}^{2} \leq \mathscr{V}_{(\mathscr{S})}(v, v) \leq C_{1}\|v\|_{-1 / 2, \mathscr{S}}^{2} \quad \text { for all } v \in H^{-1 / 2}(\mathscr{S}),
$$

where $\mathscr{V}_{(\mathscr{S})}$ is defined by

$$
\mathscr{V}_{(\mathscr{S})}(u, v)=\int_{\mathscr{S}} \int_{\mathscr{S}} \frac{u\left(s_{1}\right) v\left(s_{2}\right)}{\left|s_{1}-s_{2}\right|} d s_{1} d s_{2}
$$

In addition, they also showed that the operator $\mathscr{V}_{(\mathscr{S})}$ defined by

$$
\left(\mathscr{V}_{(\mathscr{S})} u\right)\left(s_{2}\right)=\int_{\mathscr{S}} \frac{u\left(s_{1}\right)}{\left|s_{1}-s_{2}\right|} d s_{1}
$$


is an isomorphism of $H^{s}(\mathscr{S})$ onto $H^{s+1}(\mathscr{S})$ for all real $s$. This implies that there are constants $C_{0}$ and $C_{1}$ such that

$$
C_{0}\|w\|_{s, \mathscr{S}} \leq\left\|\mathscr{V}_{(\mathscr{S})} w\right\|_{s+1, \mathscr{S}} \leq C_{1}\|w\|_{s, \mathscr{S}} \quad \text { for all } w \in H^{s}(\mathscr{S}) .
$$

The above norms denote the norms in $H^{s}(\mathscr{S})$ and $H^{s+1}(\mathscr{S})$.

Remark 5.1. The inequalities (5.1) hold for $\Omega=\mathscr{S}$ when $\mathscr{S}$ consists of the union of polygonal faces and is the boundary of a region in $R^{3}$. Consequently, we can apply the results of $\S 4$ in this case (see Remark 4.2).

The plane domain $\Omega$ can be extended to be the smooth boundary of a bounded domain in $R^{3}$. We shall denote this extended surface by $\mathscr{S}$. The following lemma will be critical in the analysis provided in the remainder of this section. Its proof will be given at the end of this section.

Lemma 5.1. Let $\sigma \in L^{2}(\Omega)$ and $\tilde{\sigma}$ denote the extension by zero of $\sigma$ to $L^{2}(\mathscr{S})$. There exist positive constants $C_{2}$ and $C_{3}$ such that for $s \in[-1,0]$,

$$
C_{2}\|\sigma\|_{s} \leq\|\tilde{\sigma}\|_{s, \mathscr{S}} \leq C_{3}\|\sigma\|_{s} \quad \text { for all } \sigma \in H^{s}(\Omega) \text {. }
$$

By $(5.1), \mathscr{V}(\sigma, \sigma)=\mathscr{V}_{(\mathscr{S})}(\tilde{\sigma}, \tilde{\sigma})$ is equivalent to $\|\tilde{\sigma}\|_{-1 / 2, \mathscr{S}}^{2}$. But by Lemma 5.1, $\|\tilde{\sigma}\|_{-1 / 2, \mathscr{S}}$ is equivalent to $\|\sigma\|_{-1 / 2}$, and hence (1.1) holds.

We next verify inequality (3.5) of [4]. For our application, this translates into proving the inequality

$$
\lambda_{k}^{-1}\left\|\mathscr{V}_{k} v\right\|_{-1}^{2} \leq\left(\tilde{C} \epsilon^{k-l}\right)^{2} \mathscr{V}(v, v) \quad \text { for all } v \in \mathscr{M}_{l} .
$$

The above inequality must be proved for $l \leq k$ and some $\epsilon<1$ not depending on $l, k$, or $j$.

Let $v$ be an arbitrary function in $\mathscr{M}_{l}$. We first note that

$$
\begin{aligned}
\left\|\mathscr{V}_{k} v\right\|_{-1}^{2} & =\left\langle\mathscr{V}_{k} v, \mathscr{V}_{k} v\right\rangle_{-1}=\mathscr{V}\left(v, \mathscr{V}_{k} v\right) \\
& =\left(\mathscr{V}_{(\mathscr{S})} \tilde{v}, \widetilde{\mathscr{V}_{k} v}\right)_{\mathscr{S}} \leq\left\|\mathscr{V}_{(\mathscr{S})} \tilde{v}\right\|_{1, \mathscr{S}}\left\|\widetilde{\mathscr{V}_{k} v}\right\|_{-1, \mathscr{S}},
\end{aligned}
$$

where $(\cdot, \cdot)_{\mathscr{S}}$ is the inner product in $L^{2}(\mathscr{S})$. Applying (5.2) and Lemma 5.1 gives

and hence

$$
\left\|\mathscr{V}_{k} v\right\|_{-1}^{2} \leq C\|v\|\left\|\mathscr{V}_{k} v\right\|_{-1}
$$

$$
\left\|\mathscr{V}_{k} v\right\|_{-1}^{2} \leq C\|v\|^{2}
$$

The inverse inequality

$$
\|u\|^{2} \leq C h_{l}^{-1}\|u\|_{-1 / 2}^{2} \quad \text { for all } u \in \mathscr{M}_{l}
$$

easily follows from standard inverse inequalities, convexity and duality in the case when $\mathscr{M}_{l}$ consists of continuous piecewise linear functions. In the case of discontinuous constants, (5.5) still holds. This can be seen by noting that for $\theta \in \mathscr{M}_{l}$, the function $\boldsymbol{\theta}$ constructed in the proof of Lemma 3.2 satisfies the inequality

By convexity and (3.21),

$$
\|\theta\| \leq C\|\theta\| .
$$

$$
\|\Theta\|_{1 / 2} \leq C h_{l}^{-1 / 2}\|\theta\| .
$$


Consequently, for $u \in \mathscr{M}_{l}$,

$$
\|u\|=\sup _{\theta \in \mathscr{M}_{l}} \frac{(u, \boldsymbol{\theta})}{\|\theta\|} \leq C h_{l}^{-1 / 2} \sup _{\theta \in \mathscr{M}_{l}} \frac{(u, \theta)}{\|\Theta\|_{1 / 2}} \leq C h_{l}^{-1 / 2}\|u\|_{-1 / 2} .
$$

Combining (5.4) and (5.5) gives

$$
\lambda_{k}^{-1}\left\|\mathscr{V}_{k} v\right\|_{-1}^{2} \leq C\left(\lambda_{k} h_{l}\right)^{-1} \mathscr{V}(v, v) \quad \text { for all } v \in \mathscr{M}_{l} .
$$

The proof of (5.3) will be complete once we show that

$$
\lambda_{l}^{-1} \leq C h_{l} \text {. }
$$

Let $\mathscr{Q}_{l}$ be as in Lemmas 4.1 and 4.2. In either case, it was shown that $\mathscr{Q}_{l}$ was simultaneously bounded as an operator on both $H^{-1}(\Omega)$ and $L^{2}(\Omega)$ (with bounds which are independent of $l$ ). Thus, it follows from (3.2), Lemmas 3.1 and 3.2 that

$$
\begin{aligned}
\left(\mathscr{Q}_{l} w, \mathscr{Q}_{l} w\right)_{l}^{1 / 2} \leq C\|w\| & \text { for all } w \in L^{2}(\Omega), \\
\left(A_{l}^{-1} \mathscr{Q}_{l} w, \mathscr{Q}_{l} w\right)_{l}^{1 / 2} \leq C\|w\|_{-1} & \text { for all } w \in H^{-1}(\Omega) .
\end{aligned}
$$

Since $A_{l}$ is positive definite and symmetric with respect to $(\cdot, \cdot)_{l}$, its powers define a Hilbert scale. Using the real method of interpolation gives

$$
\left(A_{l}^{-1 / 2} \mathscr{Q}_{l} w, \mathscr{Q}_{l} w\right)_{l}^{1 / 2} \leq C\|w\|_{-1 / 2} \quad \text { for all } w \in H^{-1 / 2}(\Omega) .
$$

We then have by (1.1) and Lemmas 3.1 and 3.2,

$$
\begin{aligned}
\lambda_{l} & =\sup _{\theta \in \mathscr{M}_{l}} \frac{\mathscr{V}(\theta, \theta)}{[\theta, \theta]_{l}} \\
& \geq C \sup _{\theta \in \mathscr{M}_{l}} \frac{\left(A_{l}^{-1 / 2} \theta, \theta\right)_{l}}{\left(A_{l}^{-1} \theta, \theta\right)_{l}}=C \sup _{\theta \in \mathscr{M}_{l}} \frac{\left(A_{l}^{1 / 2} \theta, \theta\right)_{l}}{(\theta, \theta)_{l}} \geq C h_{l}^{-1} .
\end{aligned}
$$

The last inequality above follows easily from the fact that the largest eigenvalue of $A_{l}$ is bounded from below by $c h_{l}^{-2}$. This completes the proof of (5.3).

The second condition which one must verify before applying [4] is as follows. We must show that there is a constant $C_{0}$ not depending on $j$ and satisfying

$$
\mathscr{V}(v, v) \leq C_{0}\left[\mathscr{V}\left(P_{0} v, v\right)+\sum_{k=1}^{j} \lambda_{k}^{-1}\left\|\mathscr{V}_{k} P_{k} v\right\|_{-1}^{2}\right] \quad \text { for all } v \in \mathscr{M}_{j} .
$$

Let $\hat{P}_{k}$ denote the orthogonal projection operator onto the subspace $\mathscr{M}_{k}$ with respect to the inner product $\langle\cdot, \cdot\rangle_{-1 / 2}$. Lemma 3.1 of [4] shows that (5.7) will follow if we prove the analogous inequality for the equivalent form $\langle\cdot, \cdot\rangle_{-1 / 2}$, i.e., (5.7) follows from

$$
\langle v, v\rangle_{-1 / 2} \leq C\left[\left\langle\hat{P}_{0} v, v\right\rangle_{-1 / 2}+\sum_{k=1}^{j} \lambda_{k}^{-1}\left\|\hat{\mathscr{V}}_{k} \hat{P}_{k} v\right\|_{-1}^{2}\right] \quad \text { for all } v \in \mathscr{M}_{j}
$$

Here, $\hat{\mathscr{V}}_{k}: \mathscr{M}_{k} \mapsto \mathscr{M}_{k}$ is defined by

$$
\left\langle\hat{\mathscr{V}}_{k} v, \theta\right\rangle_{-1}=\langle v, \theta\rangle_{-1 / 2} \quad \text { for all } \theta \in \mathscr{M}_{k} \text {. }
$$


By Remark 3.1 of [4], inequality (5.8) will follow if we prove full regularity and approximation for $\hat{P}_{k}$. More precisely, it suffices to show that there exists a constant $C$ not depending on $k=1,2, \ldots, j$ satisfying

$$
\left\langle\left(I-\hat{P}_{k-1}\right) v, v\right\rangle_{-1 / 2} \leq C \lambda_{k}^{-1}\left\|\hat{\mathscr{V}}_{k} v\right\|_{-1}^{2} \quad \text { for all } v \in \mathscr{M}_{k} .
$$

It is well known that the spaces $H^{s}(\Omega)$ form a Hilbert scale, and hence there is an unbounded selfadjoint operator $\hat{\mathscr{V}}$ defined on $H^{-1}(\Omega)$ (with domain $L^{2}(\Omega)$ ) such that for $\gamma \in[0,1]$,

$$
\|w\|_{-1+\gamma}=\left\|\hat{\mathscr{V}}^{\gamma} w\right\|_{-1} \quad \text { for all } w \in H^{-1+\gamma}(\Omega) .
$$

Let $w$ be an arbitrary function in $H^{-1 / 2}(\Omega)$. It follows that

$$
\begin{aligned}
\left\|\left(I-\hat{P}_{k-1}\right) w\right\|_{-1} & =\sup _{\phi \in L^{2}(\Omega)} \frac{\left(\hat{\mathscr{V}}^{-1}\left(I-\hat{P}_{k-1}\right) w, \phi\right)}{\|\phi\|} \\
& =\sup _{\phi \in L^{2}(\Omega)} \frac{\left\langle\left(I-\hat{P}_{k-1}\right) w,\left(I-\hat{P}_{k-1}\right) \phi\right\rangle_{-1 / 2}}{\|\phi\|} .
\end{aligned}
$$

By convexity, the $L^{2}(\Omega)$ boundedness of $Q_{k-1},(4.3)$ and (4.5),

$$
\left\|\left(I-Q_{k-1}\right) \phi\right\|_{-1 / 2} \leq C h_{k}^{1 / 2}\|\phi\| \quad \text { for all } \phi \in L^{2}(\Omega) .
$$

Using the minimization property of the orthogonal projector gives

$$
\begin{aligned}
\left\langle\left(I-\hat{P}_{k-1}\right) \phi, \phi\right\rangle_{-1 / 2} & \leq\left\langle\left(I-Q_{k-1}\right) \phi,\left(I-Q_{k-1}\right) \phi\right\rangle_{-1 / 2} \\
& \leq C h_{k}\|\phi\|^{2} \quad \text { for all } \phi \in L^{2}(\Omega) .
\end{aligned}
$$

Combining (5.11) and (5.12) with the inequality $h_{k} \leq C \lambda_{k}^{-1}$ gives

$$
\left\|\left(I-\hat{P}_{k-1}\right) w\right\|_{-1} \leq C \lambda_{k}^{-1 / 2}\left\langle\left(I-\hat{P}_{k-1}\right) w, w\right\rangle_{-1 / 2}^{1 / 2} \text {. }
$$

Hence, for $v \in \mathscr{M}_{k}$,

$$
\begin{aligned}
\left\langle\left(I-\hat{P}_{k-1}\right) v, v\right\rangle_{-1 / 2} & =\left\langle\left(I-\hat{P}_{k-1}\right) v, \hat{\mathscr{V}}_{k} v\right\rangle_{-1} \\
& \leq C \lambda_{k}^{-1 / 2}\left\|\hat{\mathscr{V}}_{k} v\right\|_{-1}\left\langle\left(I-\hat{P}_{k-1}\right) v, v\right\rangle_{-1 / 2}^{1 / 2} .
\end{aligned}
$$

The inequality (5.10) immediately follows. This completes the proof of (5.7).

The following theorem is a consequence of (5.3) and (5.7) [4].

Theorem 5.1. Let $B_{j}$ be defined by Algorithm 2.1 with $\mathscr{V}$ defined by (1.3). Then

$$
0 \leq \mathscr{V}\left(\left(I-B_{j} \mathscr{V}_{j}\right) v, v\right) \leq \delta \mathscr{V}(v, v) \quad \text { for all } v \in \mathscr{M}_{j},
$$

where $\delta<1$ is a constant independent of $j$. In terms of matrices,

$$
0 \leq\left(\overline{\mathscr{V}}_{j}\left(I-\bar{B}_{j} \overline{\mathscr{V}}_{j}\right) \bar{v}\right) \cdot \bar{v} \leq \delta\left(\overline{\mathscr{V}}_{j} \bar{v}\right) \cdot \bar{v} \quad \text { for all } \bar{v} \in R^{n_{k}} .
$$

Remark 5.2. Additive versions of the multigrid algorithm can also be defined (as developed in [4]). Theorem 3.1 of [4] guarantees that the additive version will lead to a preconditioned system which has a condition number which is independent of the number of levels. 
Remark 5.3. We proved full regularity and approximation for the equivalent quadratic form $\langle\cdot, \cdot\rangle_{-1 / 2}$ as part of the proof of (5.7). In contrast, it is unlikely that the original form $\mathscr{V}(\cdot, \cdot)$ satisfies the full regularity and approximation condition (with constants that are independent of $j$ ).

We conclude this section with the proof of Lemma 5.1. Let $\sigma$ be in $H^{-1}(\Omega)$. We first prove the upper inequality. This is obvious for $s=0$. Moreover,

$$
\begin{aligned}
\|\tilde{\sigma}\|_{-1, \mathscr{S}} & =\sup _{\phi \in H^{1}(\mathscr{S})} \frac{(\tilde{\sigma}, \phi)_{\mathscr{S}}}{\|\phi\|_{1, \mathscr{S}}} \\
& \leq \sup _{\phi \in H^{1}(\mathscr{S})} \frac{(\sigma, \phi)}{\|\phi\|_{1}} \leq\|\sigma\|_{-1} .
\end{aligned}
$$

The upper inequality follows by interpolation.

To prove the lower inequality of the lemma, we use a simultaneously bounded extension operator $E: H^{s}(\Omega) \mapsto H^{s}(\mathscr{S})$, for $s \in[0,1]$. The existence of such an extension is well known (cf. [14]). Then,

$$
\begin{aligned}
\|\sigma\|_{-s} & =\sup _{\phi \in H^{s}(\Omega)} \frac{(\sigma, \phi)}{\|\phi\|_{s}} \\
& \leq C \sup _{\phi \in H^{s}(\Omega)} \frac{(\tilde{\sigma}, E \phi) \mathscr{S}}{\|E \phi\|_{s, \mathscr{S}}} \leq C\|\tilde{\sigma}\|_{-s, \mathscr{S}} .
\end{aligned}
$$

This completes the proof of Lemma 5.1.

\section{NUMERICAL COMPUTATIONS}

In this section, we provide the results of numerical examples illustrating the theory developed in earlier sections. We shall consider the integral equation (1.3) defined on $\Omega=[-1,1] \times[-1,1]$.

We consider the case when the subspaces are given by piecewise constant functions on a rectangular mesh. Let $m_{k}=2^{k+1}$ and define the $k$ th mesh by partitioning the domain $\Omega$ into $m_{k} \times m_{k}$ square subdomains of side length $1 / m_{k}$. The approximation space $\mathscr{M}_{k}$ is defined to be the set of functions which are picewise constant with respect to this mesh. Equations involving $\bar{B}_{0}$ are solved exactly.

Because of the fact that the mesh lines are parallel to the $x$ and $y$ axes, the integrals required for the entries of the matrix $\overline{\mathscr{V}}_{k}$ can be computed analytically. Moreover, since $\overline{\mathscr{V}}_{k}$ is translationally invariant, its action can be computed in $O\left(k n_{k}\right)$ operations by use of the fast discrete Fourier transform [6].

We will present results using the multigrid operator as a preconditioner in a preconditioned conjugate gradient iteration. One factor which can be used to interpret the efficiency of the proposed iterative scheme is the number of iterations required to achieve a certain accuracy. Let NI be the number of steps required to reduce the initial error by the factor of $10^{-6}$, i.e.,

$$
\left\|\bar{e}_{N I}\right\| \leq 10^{-6}\left\|\bar{e}_{0}\right\| \text {. }
$$

Here, $\bar{e}_{i}=\bar{u}-\bar{v}_{i}$, where $\bar{u}$ is the solution of (3.23) and $\bar{v}_{i}$ is the ith iterate in the iterative algorithm.

We compare two iterative schemes for computing the solution of (3.23). The first is the conjugate gradient algorithm using the multigrid preconditioner of Algorithm 3.1. The second is the conjugate gradient algorithm (CG) applied 
TABLE 6.1. CG preconditioned with $\bar{B}_{j}$

\begin{tabular}{|c|c|c|}
\hline $1 / h$ & $\kappa\left(\bar{B}_{j} \overline{\mathscr{V}}_{j}\right)$ & NI \\
\hline 16 & 1.92 & 7 \\
32 & 1.98 & 8 \\
64 & 2.00 & 8 \\
128 & 2.01 & 8 \\
256 & 2.01 & 8 \\
\hline
\end{tabular}

TABLE 6.2. CG directly applied to (3.23)

\begin{tabular}{|c|c|c|}
\hline $1 / h$ & $\kappa\left(\overline{\mathscr{V}_{j}}\right)$ & $\mathrm{NI}$ \\
\hline 16 & 44.3 & 18 \\
32 & 89.0 & 26 \\
64 & 176.9 & 37 \\
128 & 344.9 & 51 \\
256 & 657.0 & 68 \\
\hline
\end{tabular}

directly to (3.23). In Tables 6.1 and 6.2, we report the condition numbers, $\kappa\left(\bar{B}_{j} \overline{\mathscr{V}}_{j}\right)$ and $\kappa\left(\overline{\mathscr{V}}_{j}\right)$, respectively, and the number of iterative steps required to satisfy the condition (6.1). The use of the multigrid preconditioner results in significant improvements in both the condition number as well as the number of iterations required to satisfy (6.1). Note the condition numbers in Table 6.1 appear bounded. This is in agreement with the theoretical results of Theorem 5.1 .

\section{BIBLIOGRAPHY}

1. R.E. Bank and T. Dupont, An optimal order process for solving finite element equations, Math. Comp. 36 (1981), 35-51.

2. D. Braess and W. Hackbusch, $A$ new convergence proof for the multigrid method including the V-cycle, SIAM J. Numer. Anal. 20 (1983), 967-975.

3. J.H. Bramble and J.E. Pasciak, New convergence estimates for multigrid algorithms, Math. Comp. 49 (1987), 311-329.

4. 447-471.

5. __ The analysis of smoothers for multigrid algorithms, Math. Comp. 58 (1992), 467-488.

6. __ An efficient numerical procedure for the computation of steady state harmonic currents in flat plates, IEEE Trans. on Mag. Mag-19 (1983), 2409-2412.

7. J.H. Bramble, J.E. Pasciak, J. Wang, and J. Xu, Convergence estimates for multigrid algorithms without regularity assumptions, Math. Comp. 57 (1991), 23-45.

8. J.H. Bramble, J.E. Pasciak and J. Xu, The analysis of multigrid algorithms with nonnested spaces or noninherited quadratic forms, Math. Comp. 56 (1991), 1-34.

9. __ Parallel multilevel preconditioners, Math. Comp. 55 (1990), 1-22.

10. J.H. Bramble and J. Xu, Some estimates for weighted $L^{2}$ projections, Math. Comp. 56 (1991), 463-476. 
11. A. Brandt, Multi-level adaptive solutions to boundary-value problems, Math. Comp. 31 (1977), 333-390.

12. A. Brandt and A.A. Lubrecht, Multilevel matrix multiplication and fast solution of integral equations, J. Comput. Phys. 90 (1990), 348-370.

13. P.L. Butzer and H. Berens, Semi-groups of operators and approximation, Springer-Verlag, New York, 1967.

14. P. Grisvard, Elliptic problems in non smooth domains, Pitman, Boston, 1985.

15. W. Hackbusch, Multi-grid methods and applications, Springer-Verlag, New York, 1985.

16. J. F. Maitre and F. Musy, Algebraic formalization of the multigrid method in the symmetric and positive definite case-a convergence estimation for the V-cycle, Multigrid Methods for Integral and Differential Equations (D. J. Paddon and H. Holstien, eds.), Clarendon Press, Oxford, 1985.

17. J. Mandel, S. McCormick, and R. Bank, Variational multigrid theory, Multigrid Methods (S. McCormick, ed.), SIAM, Philadelphia, PA, 1987, pp. 131-178.

18. J.C. Nédélec and J. Planchard, Une méthode variationnelle d'éléments finis pour la résolution numérique d'un problème extérieur dans $R^{3}$, RAIRO Sér. Rouge 7 (1973), 105-129.

19. T. von Petersdorff and E.P. Stephan, On the convergence of the multigrid method for a hypersingular integral equation of the first kind, Numer. Math. 57 (1990), 379-391.

Department of Mathematics, Cornell University, Ithaca, New York 14853

E-mail address: bramble@math. cornell.edu

Statistics Research Section, Australian National University, Canberra, ACT 2601, Australia

E-mail address: zleyk@vila.anu.edu.au

Department of Applied Science, Brookhaven National Laboratory, Upton, New York 11973

E-mail address: pasciak@bnl.gov 